\title{
Versorgungsforschung - braucht es das in der Schweiz?
}

\section{Thomas Rosemann}

Direktor des Instituts für Hausarztmedizin UniversitätsSpital Zürich

Korrespondenz:

Prof. Dr. med.

Thomas Rosemann PhD Institut für Hausarztmedizin UniversitätsSpital Zürich Pestalozzistrasse 24

CH-8091 Zürich

Tel. 0442559099

Fax 0442559855

thomas.rosemann[at]usz.ch

\section{Chronische Krankheiten, Multimorbidität und Fragmentierung des Gesundheitssystems} Die Entwicklung der Medizin in den letzten hundert Jahren ist eine grosse Erfolgsgeschichte: Jahrhundertelange Geisseln der Menschheit, die die Lebensqualität und mehr noch die Lebenserwartung oft stark limitiert haben, wurden durch immense pharmakologische und medizintechnische Entwicklungen beherrschbar: angefangen bei Impfungen und Antibiotika, die epidemiologisch unzweifelhaft zu den segensreichsten Entdeckungen überhaupt zählen, über eine Fülle von Medikamenten, die etwa kardiovaskuläre Erkrankungen therapierbar machten, bis hin zu modernsten interventionellen Techniken in der Chirurgie und Kardiologie.

All dies ermöglicht uns heute eine nie erreichte Lebenserwartung, aber eben auch das Erleben einer Ansammlung chronischer Erkrankungen und schafft damit ganz neue Herausforderungen für die Versorgung. Diese wiederum wird durch den enormen Fortschritt, der zu immer neuen Subspezialitäten führt, immer komplexer und fragmentierter. Hinzu kommt noch eine weitere Folge der ungeahnten medizinischen Möglichkeiten: Alle kosten Geld, und auch dies ist nur begrenzt im System vorhanden. $\mathrm{Zu}$ den medizinischen Fragestellungen kommen somit ökonomische und das Problem, wie Versorgung optimal zu gestalten sei.

Im Schweizer Gesundheitssystem werden aktuell Massnahmen heftig diskutiert, die auf übergeordneter Ebene Versorgung strukturieren sollen, allen voran «Managed Care» und «Integrierte Versorgung». Diese Diskussion wird aber auf weiten Strecken politisch geführt, anstatt auf Basis valider gesundheitswissenschaftlicher Erkenntnisse. Mit etwas Distanz betrachtet, wirkt es fast ein wenig befremdlich, dass es einerseits eine Fülle von Studien mit Tausenden Patienten gibt, bei denen unterschiedliche Pharmaka einer Substanzklasse miteinander verglichen werden, um dann einen oft minimen Vorteil für eine Substanz herauszuschälen. Und auf der anderen Seite werden Eingriffe in das Gesundheitssystem weitgehend willkürlich und ohne belastbare Erkenntnisse über ihre Auswirkungen implementiert. Neben den bereits erwähnten Massnahmen wären die «Diagnostic Related Groups» (DRG) oder auch der «Skillmix», also die Frage, welche Professionen in welchem Masse in die Behandlung involviert sein sollen, oder auch die Selbstdispensation zu nennen. Diese Massnahmen werden leidenschaftlich diskutiert, aber es liegen keine harten Fakten vor, ja mehr noch, es gibt noch

\section{La recherche sur les soins (Health Services Research) est-elle utile en Suisse?}

Les nouveaux défis qui se posent en raison du vieillissement de la population ainsi que les maladies chroniques et la polymorbidité qui en découlent ne peuvent pas uniquement être résolus par le recours à de nouveaux médicaments ou à de nouvelles techniques. Il s'agit bien plus de mettre en place une organisation optimale des soins et de coopérer avec les fournisseurs de prestations. La recherche sur les soins (Health Services Research, HSR) traite justement de l'organisation de ceux-ci. Ce domaine, en plein essor sur le plan international, est bien implanté aux Etats-Unis et dans de nombreux pays d'Europe et fait figure de troisième pilier aux côtés de la recherche fondamentale et de la recherche clinique. La recherche sur les soins vise l'obtention de résultats importants pour les patients et se différencie de la recherche clinique et des études clinicopharmacologiques, car elle prend en compte les conditions réelles et les aspects économiques.

La recherche sur les soins est un champ pluridisciplinaire qui porte tant sur des disciplines où l'on pratique la recherche à laquelle participent des cliniciens, des épidémiologistes, des sociologues et des psychologues que sur les méthodes de recherche. Hormis les analyses épidémiologiques, on intègre aussi l'analyse qualitative, les modèles quantitatif et ceux permettant de générer ou de vérifier des hypothèses. Dans de nombreux pays, la recherche sur les soins est largement subventionnée. En Suisse toutefois, elle n'en est qu'à ses débuts et sa promotion n'est possible qu'au travers du nouveau programme de l'Académie Suisse des Sciences Médicales.

nicht einmal Bestrebungen, diese zu generieren. Hintergrund ist, dass der Forschungszweig, der sich mit diesen Fragestellungen wissenschaftlich auseinandersetzt, in der Schweiz aktuell praktisch nicht entwickelt ist: die Versorgungsforschung. 


\section{Definition}

Es existieren verschiedene Definitionen der Versorgungsforschung oder «Health Services Research» (HSR). Im deutschsprachigen Raum am weitesten verbreitet sind die Definitionen von Pfaff, Schrappe und der Deutschen Bundesärztekammer [1, 2]. Am prägnantesten bringt es allerdings die Definition der amerikanischen «Agency for Healthcare Research and Quality» (AHQR) zum Ausdruck:

«Health Services Research examines how people get access to health care, how much care costs and what happens to patients as a result of this care. The main goal of HSR is to identify the most effective way to organize, manage, finance, and deliver highquality care, reduce medical errors and improve patient safety» [3].
Versorgungsforschung zielt also auf die patientenrelevanten Ergebnisse der Versorgung und dies im Unterschied zu klinischen und/oder klinischpharmakologischen Studien - unter realen Bedingungen und Berücksichtigung ökonomischer Aspekte. Sie ist Mittler zwischen klinischer Forschung und täglicher Praxis. Ein alltägliches Phänomen in der medizinischen Versorgung ist die Kluft («gap») zwischen den Erkenntnissen aus der klinischen Forschung, die primär nur die «efficacy» (Wirksamkeit unter Idealbedingungen) untersucht, und der «effectiveness», also der Wirkung unter realen Bedingungen. Diese Kluft wird wissenschaftlich im «Throughput Modell», das zentral in der Versorgungsforschung ist, abgebildet.

\section{Versorgungsforschung zielt auf die patientenrelevanten Ergebnisse der Versorgung unter realen Bedingungen und Berücksichtigung ökonomischer Aspekte.}

In dieser Definition wird bereits deutlich, dass HSR auf das Ergebnis, also den «Outcome», fokussiert ist, daher hat sich in jüngerer Zeit auch der Ausdruck «Outcome Research» als Synonym etabliert. In der Definition von Clancy und Eisenberg, 1998 in Science publiziert, werden zusätzlich soziale Faktoren und Wertvorstellungen des Patienten betont

«Outcomes research - the study of the end results of health care services that takes patients' experiences, preferences and values into account» [4].

\section{Methodik}

\section{Multidisziplinarität}

Versorgungsforschung ist ein multidisziplinäres Forschungsfeld sowohl in Bezug auf die forschenden Disziplinen, vornehmlich Kliniker, Epidemiologen, Sozialwissenschaftler und Psychologen, als auch in Bezug auf die Forschungsmethoden: Neben epidemiologischen Analysen, die oftmals vorhandene Datenbestände etwa von Versicherern oder aus

\section{Abbildung 1}

Das systemtheoretische Modell des Versorgungssystems, «Throughput Modell» (nach Pfaff [1]).

\begin{tabular}{l}
\hline \multicolumn{1}{|c}{ Input } \\
\hline Im Kontext des Inputs \\
stehen bei den «Ge- \\
sundheitsdienstleis- \\
tern» vor allem Fragen \\
der unterschiedlichen \\
Professionen und ih- \\
rer optimalen Koopera- \\
tion unter Berücksichti- \\
gung der individuellen \\
Sozialisation im Vorder- \\
grund. Unter den «Res- \\
sourcen» werden hier \\
insbesondere materielle \\
Ressourcen verstanden. \\
Auf Patientenebene \\
ergeben sich Frage- \\
stellungen im Hinblick \\
auf den Bedarf, die \\
Nachfrage, den Zugang \\
und die Inanspruch- \\
nahme.
\end{tabular}
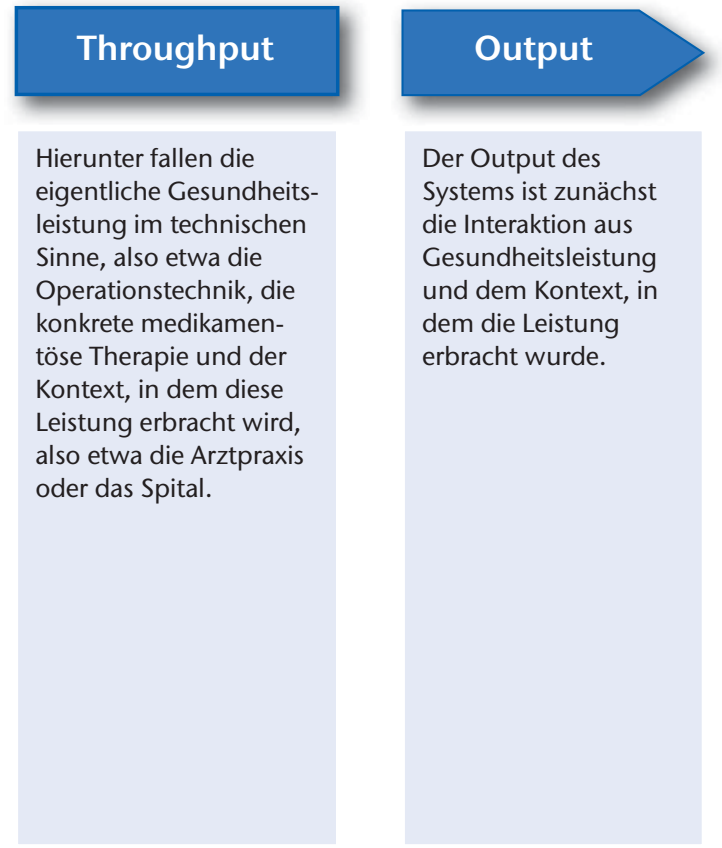

\section{Outcome}

Insgesamt besteht die Aufgabe des Gesundheitssystems abe darin, aus den InputFaktoren mit Hilfe von Versorgungsleistung einen Output zu erzeugen, der eine positive Auswirkung auf den Patienten hat (Outcome). Dieser Nutzen kann auf körperlicher Ebene (z. B. Überlebensrate), psychischer (krankheitsbezogene Lebensqualität) oder auch auf anderen Ebenen erfolgen (Verhaltensebene, soziale Ebene, kulturelle oder spirituelle Ebene). 
öffentlichen Registern nutzen, kommen auch qualitative, also hypothesengenerierende, und natürlich quantitative, also hypothesenprüfende Designs zum Einsatz. Häufig bauen diese auch dergestalt aufeinander auf, dass zunächst «facilitators» und «barriers» einer gewünschten Veränderungen qualitativ erfasst werden, dann eine Intervention massgeschneidert wird und deren Implementierung dann in einer (Cluster-)randomisierten, kontrollierten Interventionsstudie untersucht wird. Methodisch ist Versorgungsforschung sehr anspruchsvoll, da unter «Reallife»-Bedingungen viele Einflussgrössen, die in klinischen Studien mit hochselektierten Patienten ausgeklammert werden können, nicht ausgeschlossen werden können, sondern erfasst und in der Auswertung berücksichtigt werden müssen.

Klassische Gebiete der Versorgungsforschung sind unter anderem [5]:

- Epidemiologie (Beobachtungs- und Kohortenstudien)

- Evidence Based Medicine (Übertragbarkeit auf die reale Versorgung)

- Qualitäts- und Patientensicherheitsforschung

- Lebensqualitätsforschung (Lebensqualität als wichtigster Bestandteil der «Patient Reported Outcomes», PROs)

- Pflegeforschung

- Gesundheitsökonomie

- Public Health

- Ethik (Auswirkung auf Patienten und Versorger)

- Implementierungsforschung (Wie werden Interventionen optimal in die Routineversorgung implementiert?).

\section{Historie und internationale Situation}

\section{USA}

Versorgungsforschung hat ihre Wurzeln in den Vereinigten Staaten und reicht mehr als 60 Jahre zurück. In den 60er Jahren wurde der Begriff «Health Services Research» etabliert, ein «National Center for Health Services Research and Development» gegrün-

\section{Grossbritannien}

Seit den 80er Jahren des letzten Jahrhunderts wird HSR in Grossbritannien durch verschiedene Institutionen finanziell gefördert. Seit 2005 existiert das «Health Services Research Network», das u.a. durch den «National Health Service» (NHS) finanziert wird. Die Versorgungsforschung ist in Grossbritannien sehr anwendungsorientiert, was in der aktuellen Definition der NHS Confederation zum Ausdruck gebracht wird: «all research that underpins improvements in the way health services are financed, organized, planned and delivered, and includes health technology assessments and health policy research» [8]. Ein grosser Forschungsbereich in Grossbritannien ist insbesondere das «Pay-forperformance»-Programm, das eine qualitätsorientierte Vergütung für Hausärzte etablierte und zu einer deutlichen Einkommenssteigerung der englischen Hausärzte führte.

\section{Deutschland}

1989 wurde in Deutschland ein Förderprogramm «Gesundheitswissenschaften Public Health» aufgelegt, das als Anschubfinanzierung für Versorgungsforschung wirkte. Der Sachverständigenrat der Bundesregierung hat wiederholt explizit die Versorgungsforschung angemahnt, indem er feststellte (2001):

«Die belastbare Evidenz (...) zur Über-, Unterund Fehlversorgung ist vor allem deshalb so gering, weil im Bereich der Universitäten ein völlig unangemessenes Missverhältnis zwischen klinischer Forschung und Versorgungsforschung zu verzeichnen ist (...). Damit verbunden ist eine massive Überschätzung und Überbewertung der medizinischen Interventionen für die Lebenserwartung und Lebensqualität und eine nicht zukunftsfähige Vernachlässigung von Forschung zur interdisziplinären und sektorenübergreifenden Gestaltung von Versorgungszusammenhängen» [9].

2002 wurde in Deutschland erstmals der Kongress für Versorgungsforschung abgehalten, und parallel folgten mehrere Förderprogramme, die die

\section{Versorgungsforschung gilt in den USA heute als dritte Säule der medizinischen Forschung.}

det und auch ein entsprechendes wissenschaftliches Journal ins Leben gerufen. Heute gilt in den USA Versorgungsforschung als dritte Säule der medizinischen Forschung, neben der biomedizinischen und der klinischen Forschung [6,7]. Das Gesundheitsdepartment hat später die «Agency for Healthcare Research and Quality» (AQHR) ins Leben gerufen, die heute hohe Beträge für Versorgungsforschungsprojekte zur Verfügung stellt [3].
Versorgungsforschung mit hohen Förderbeträgen unterstützen. Mittlerweile sind neben dem Bundesministerium für Bildung und Forschung (BMBF), das in der Wissenschaftsförderung eine vergleichbare Funktion wie der Schweizer Nationalfonds (SNF) einnimmt, auch die gesetzlichen Krankenversicherungen, die privaten Krankenversicherer sowie die deutschen Rentenversicherer an der Förderung dieser Forschung substanziell beteiligt. Als wegweisend ist 
Abbildung 2

Förderung der Versorgungsforschung durch die Bangerter Stiftung und die SAMW.

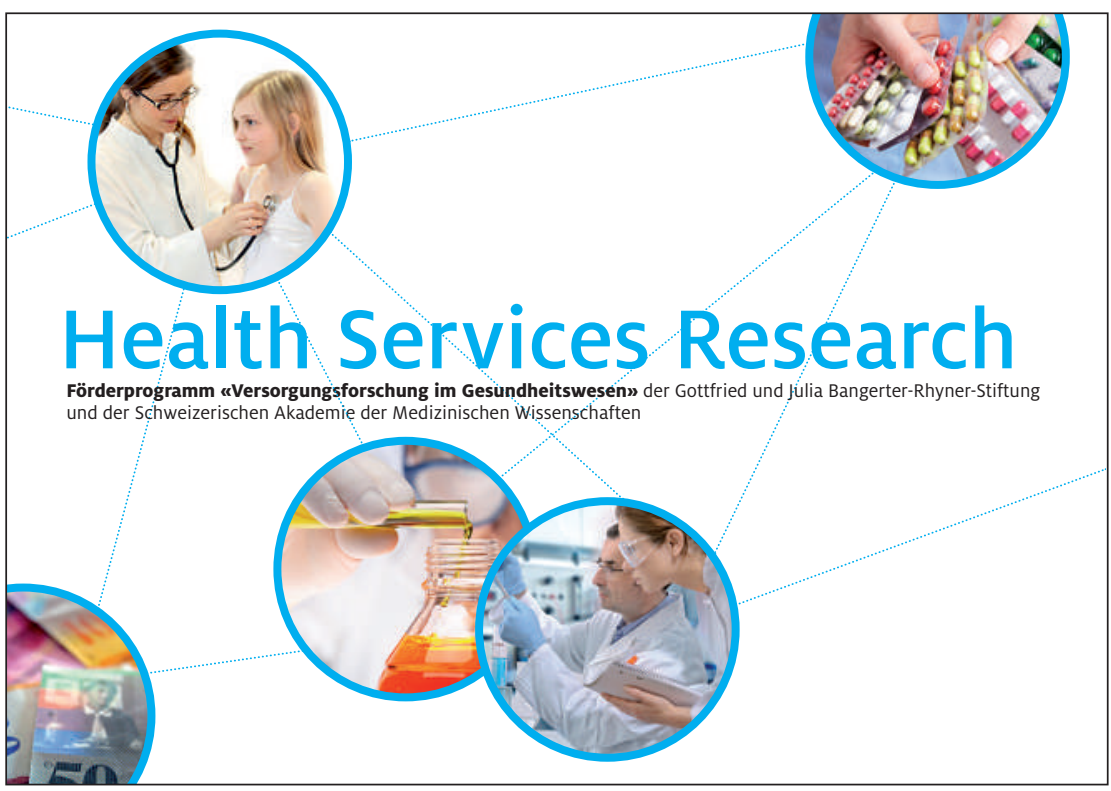

zu betrachten, dass bereits 2005 der Deutsche Ärztetag beschloss, die Versorgungsforschung durch die Bundesärztekammer aus den Mitgliederbeiträgen aller deutschen Ärzte zu fördern [10].

\section{Versorgungsforschung in der Schweiz}

In der Schweiz gibt es derzeit eine Fülle von Fragestellungen, auf die Versorgungsforschung Antworten liefern könnte [11]. Am Beispiel der DRGs wird aber deutlich, welche Chancen hier verpasst werden. Dieser nachhaltige Eingriff in das Vergütungssystem mit Auswirkungen auf fast alle Versorgungsbereiche (stationär, Rehabilitation, ambulante ärztliche Versorgung) wird ohne adäquate Begleitforschung vorgenommen. Zwar werden Auswirkungen auf das Personal in Spitälern untersucht - wozu es auch schon reichlich Daten aus anderen Ländern gibt - die Frage ob und in welchem Umfang es tatsächlich zu Verlagerungseffekten kommen wird, kann jedoch mit keinem Projekt valide beantwortet werden.

Bedauerlicherweise ist die Forschung in der Schweiz nach wie vor sehr grundlagenorientiert, Förderprogramme auf kantonaler oder nationaler Ebene fehlen völlig. Die Einrichtung eines nationalen Forschungsprogrammes wurde abgelehnt, der Nationalfonds finanziert praktisch keine Versorgungsforschung. Einzig die Novartis Schweiz AG unterstützt am Institut für Hausarztmedizin ein auf Versorgungsforschung ausgerichtetes PhD-Programm, das allerdings nur in Verbindung mit der Universität Nijmegen/NL realisiert werden konnte, da das in Zürich etablierte MD-PhD-Programm ebenfalls nur auf klinische bzw. Grundlagenforschung ausgerichtet ist. Angesichts des Stellenwerts, den sich die Versorgungsforschung durch ihre Ergebnisse international bereits erarbeitet hat, scheint es fast beabsichtigt, dass in der Schweiz gesundheitspolitische Weichen lieber «eminenzbasiert» und auf politischem Wege gestellt werden, als auf Basis valider Daten. Mittelfristig mag dies für die einzelnen Interessengruppen reizvoll sein, langfristig wird sich die Vernachlässigung einer Forschung, die das Gesamtsystem im Auge behält, aber nicht nur auf die Versorgungsqualität negativ auswirken, es wird auch finanziell desaströs enden.

Als Lichtblick ist das von der Schweizerischen Akademie der Medizinischen Wissenschaften (SAMW) und mit finanzieller Unterstützung der Bangerter Stiftung ins Leben gerufene Förderprogramm «Versorgungsforschung in der Medizin» zu werten. Obwohl hier zukünftig pro Jahr 1 Million Franken zur Verfügung stehen werden, ist der Betrag angesichts der drängenden Fragen und insbesondere in Relation zu den Hunderten Millionen, die in die anderen Forschungsbereichen investiert werden, eine kleine Summe. Es bleibt $\mathrm{zu}$ hoffen, dass die Schweiz den internationalen Weckruf hört. Ein gutes Gesundheitssystem besteht nämlich nicht nur aus exzellenten Einzelakteuren, sondern aus dem möglichst optimalen Zusammenspiel aller Akteure.

\section{Literatur}

1 Pfaff H. Versorgungsforschung - Begriffsbestimmung, Gegenstand und Aufgaben. In: Pfaff H, Schrappe K, Lauterbach W, Engelmann U, Halber M (Hrsg.). Gesundheitsversorgung und Disease Management. Grundlagen und Anwendungen der Versorgungsforschung. Bern: Verlag Hans Huber; 2003.

2 Bundesärztekammer. www.bundesaerztekammer.de/ page.asp?his=1.6.3289.3293.3294

3 AHQR. www.ahrq.gov

4 Clancy C, Eisenberg J. Outcomes Research: Measuring the end results of health care. Science. 1998;282: 245-6.

5 Pfaff H, Neugebauer, Glaeske, Schrappe K (Hrsg). Lehrbuch Versorgungsforschung, Stuttgart: Schattauer Verlag; 2011.

6 Black N. Health services reserach: saviour or chimera? Lancet. 1997;349:1834-6.

7 Lohr KN, Steinwachs DM. Health Services Research: an evolving definition of the field. Health Serv Res. 1994;27:15-7.

8 NHS Confederation. www.nhsconfed.org/Networks/ HealthServiceResearchNetwork/Pages/HSRN.aspx

9 Sachverständigenrat für die Konzertierte Aktion im Gesundheitswesen. www.svr-gesundheit.de/ Gutachten/Gutacht01/Kurzf-de.pdf

10 BMBF. Versorgungsforschung. Ergebnisse der gemeinsamen Förderung durch das BMBF und die Spitzenverbände der gesetzlichen Krankenversicherung (2005-2008) . Bonn, Berlin; 2008.

11 SAMW. www.samw.ch/de/Forschung/Versorgungsforschung.htm 